\title{
Perspectives of health practitioners and the key population on predictors of relapse in physical activity and dietary behavior: a concept mapping study
}

Eline Roordink ( $\nabla$ eline.roordink@vu.nl )

Vrije Universiteit Amsterdam https://orcid.org/0000-0002-4132-2896

Ingrid H.M. Steenhuis

Vrije Universiteit Amsterdam

Willemieke Kroeze

Vrije Universiteit Amsterdam

Mai J.M. Chinapaw

VU medisch centrum

Maartje M. van Stralen

Vrije Universiteit Amsterdam

\section{Research article}

Keywords: Relapse, dietary behavior, physical activity, concept mapping

Posted Date: August 19th, 2020

DOI: https://doi.org/10.21203/rs.3.rs-44783/v1

License: (c) (i) This work is licensed under a Creative Commons Attribution 4.0 International License.

Read Full License 


\section{Abstract \\ Background}

To prevent people from relapsing into unhealthy habits, insight into predictors of relapse in physical activity and dietary behavior is needed. Therefore, we aimed to explore predictors of relapse in physical activity and dietary behavior from the perspectives of health practitioners and the key population (i.e. adults who recently lost weight and experienced relapse).

\section{Methods}

We used concept mapping to collect data among five groups of health practitioners $(\mathrm{N}=39)$ and three key population groups $(\mathrm{N}=21)$. First participants' ideas on potential predictors were collected. Subsequently, these ideas were individually sorted by relatedness and rated on importance. Concept maps were created for each group using principal component analysis and cluster analysis.

\section{Results}

In total 43 predictors were identified, of which the majority belonged to the individual domain compared to the environmental domain. Although the majority of perceived predictors were mentioned by both stakeholder groups, both groups had different opinions regarding the importance of predictors. Also, few predictors were mentioned by all practitioner groups, but not by the key population, and vice versa. Practitioners indicated change in daily structure, stress, lack of effective coping skills, habitual behavior, and lack of self-efficacy regarding losing weight as most important recurrent (i.e. mentioned in all practitioner groups) predictors. The key population indicated lifestyle imbalance or experiencing a life event, lack of perseverance, negative emotional state, abstinence violation effect, decrease in motivation and indulgence as most important recurrent predictors.

\section{Conclusions}

Both stakeholder groups predominantly rated individual factors as most important perceived predictors of relapse. The finding that both groups differed in opinion regarding importance of predictors or identified different predictors, may provide an opportunity to enhance lifestyle coaching by ensuring it is patient-centered and tailored.

\section{Background}

For people with obesity, weight loss starting from five percent of their body weight can already have major health benefits, such as improved body composition and metabolic function (1). However, maintaining weight loss by sustainable changes in physical activity and dietary behavior has proven to 
be challenging. On average, $30-35 \%$ of the lost weight is regained in the first year after weight loss, and after this year weight gain generally continues (2). To maintain weight loss, relapse, i.e. a breakdown or failure in a person's attempt to change and maintain their target behavior, has to be prevented (3). To effectively prevent people from relapsing, insight into the predictors of relapse in physical activity and dietary behavior is needed. Therefore, the current study aims to explore predictors of relapse in physical activity and dietary behavior from the perspectives of two stakeholder groups: health practitioners who supervise and coach adults in their process of weight loss and adults who recently lost weight and experienced one or more relapses.

Insight into predictors of relapse is provided by theoretical models such as Marlatt's Relapse Prevention Model (RPM) (4) and the self-regulation theory (5). Although, the RPM was originally developed for relapse prevention in drug abstinence, it has been successfully applied to a wide range of health behaviors, including obesity $(6,7)$. According to the RPM, risk of relapse occurs when an individual enters a so-called high-risk situation, i.e. any situation that poses a threat to the individual's sense of control. When someone lacks effective coping responses while entering the high-risk situation, a decrease in selfefficacy will occur and the probability of lapse, i.e. a slip or mistake, will arise. If this person also has positive outcome expectancies towards the old habitual behavior, the chance of lapsing will increase even more (3). Whether this first lapse is followed by a complete relapse depends on the individual's attribution of the cause of the lapse. A negative attribution of the cause is called the abstinence violation effect: if the individual attributes the lapse to their own personal failure (e.g. guilt, shame) and to stable, internal factors beyond their control (e.g. no willpower), the risk of relapse increases $(3,8)$.

According to the self-regulation theory, the abstinence violation effect is one of the possible lapseactivated responses which contributes to self-regulation failure (9). Within this theory, lapse-activated responses are described as a class of behaviors that emerge after an initial failure of self-regulation, a lapse. These responses cause a minor breakdown in self-control, often activating factors that prevent the reassertion of self-control, resulting in an acceleration of the breakdown. Often it is not the lapse itself, but the subsequent breakdown in self-control that has the most severe results on behavioral maintenance $(9,10)$.

Apart from theories, insight into predictors of relapse can be obtained from previous studies. The recent literature review from Roordink et al. (11) on the predictors of lapse and relapse in physical activity and dietary behavior, based on 41 prospective studies, found that for physical activity people with a lower self-efficacy, less behavioral processes of change (i.e. covert and overt activities to modify behavior), and less self-regulation had a higher risk of relapsing (11). For dietary behavior, they found that people with lower self-efficacy had a higher risk of relapsing (11). However, the review also showed that there is still insufficient evidence for most predictors of relapse. An in-depth understanding of personal perspectives on relapse after weight loss of key stakeholders such as adults attempting weight loss themselves and health practitioners, is lacking in the current literature. 
Perspectives from these key stakeholders could provide new and important insights from daily practice on predictors of relapse in physical activity and dietary behavior, which can inform future relapse prevention interventions. Hence, we aimed to identify predictors of relapse in physical activity and dietary behavior, from the perspective of health practitioners who coach individuals in their process of weight loss and the perspective of individuals who have experienced relapses themselves, using concept mapping. Concept mapping is a structured methodology combining qualitative and quantitative methods to integrate group thought and perspectives about a particular topic, in order to yield a conceptual framework (12). Concept mapping has been applied successfully in addressing complex issues in health care (13).

\section{Methods}

\section{Design}

A concept mapping study was conducted with data collection between November 2017 and July 2018 . The following steps from the process of concept mapping $(12,14)$ were applied: 1 . preparation, 2. generation of statements, 3 . structuring of statements, 4 . representation of statements, and 5. interpretation of maps, see Fig. 1.

[Figure 1. The concept mapping process. Adapted from "An introduction to concept mapping for program planning and evaluation" by W. Trochim, 1989, Evaluation and Program Planning, 12, 1-16.]

\section{Step 1. Preparation}

Conceptualization is at its best when it includes a diversity of relevant people, to ensure that a variety of viewpoints is considered (14). Therefore, our study included two stakeholder groups: 1 . health practitioners supervising and coaching adults in losing weight, and 2. key population, adults who recently lost weight and experienced at least one relapse (determined by the key population itself). Within these stakeholder groups, we aimed for a diverse sample, e.g. multiple professions and a demographically heterogeneous key population. Inclusion criteria were: speaking Dutch and for the key population recently (<one year) lost weight through behavioral change instead of a self-reported crash diet (i.e. consuming minimal levels of food to lose a lot of weight in a short period of time) or bariatric surgery.

Per stakeholder group we aimed for four subgroups across the Netherlands, including 7-10 participants per subgroup or until saturation was reached, which is in accordance with the concept mapping literature (15). Health practitioners were recruited by a call for participation on Linkedln, an online network for professionals. Health practitioners had the possibility to sign up individually or with a group of colleagues. The key population was recruited via multiple strategies: through health practitioners participating in our study, a call for participation on social media (Facebook, Linkedln), direct contact with walking groups, and flyers at general practitioner practices, health centers and a university. Additionally, we made use of our personal network and snowball sampling. Participants received a certificate of participation (health practitioners only), a factsheet with the study findings, and a small financial 
compensation. After agreeing to participate, online informed consent was obtained. According to the Dutch Medical Research Involving Human Subjects Act, our study did not require further evaluation by the Research Ethics Review Committee.

\section{Step 2. Generation of statements}

One week before the on-site concept mapping session, in which a subgroup got together, each participant received a link to an online questionnaire per email. The questionnaire was developed for this study and contained questions on age, sex, educational level, and profession (health practitioners only), see Supplementary file 1. Based on the standard classification of the Central Bureau of Statistics Netherlands (CBS), educational level was categorized into three levels: low level of education (primary education and lower general secondary education), middle level of education (higher general secondary education, preuniversity education, and secondary vocational education), and high level of education (bachelor's degree, master's degree, and doctoral degree; (16)). Furthermore, participants were encouraged to share all their ideas on perceived predictors of relapse in physical activity and dietary behavior, by responding to the following focus statement: "A relapse in physical activity and/or dietary behavior is caused by...". The purpose of a focus statement is to give the brainstorm an area of focus, in order to address the primary research question (12). Health practitioners were asked to address the focus statement on population level, i.e. based on their professional experience, whereas the key population did this based on personal experience. All collected ideas, hereafter referred to as statements, were used as input for a group brainstorm during the on-site concept mapping session, which was facilitated by two researchers (ER with MVS or WK). During the brainstorm participants were encouraged to come up with more statements and clarification was asked for statements that were unclear, resulting in an extensive list of unique statements per subgroup.

\section{Step 3. Structuring of statements}

Following the brainstorm during the on-site concept mapping session, participants structured the statements by individually sorting the statements into piles of related statements, i.e. categories. To complete this task, health practitioners used the online software program Ariadne (17), whereas the key population received the statements on printed cards. Due to the settings of the software program, a minimum of three and a maximum of ten categories were required, with at least two statements per category. A miscellaneous category was prohibited. Participants were instructed to individually place every statement in a category and name the categories. Subsequently, each participant individually rated the importance of every statement on a 5-point Likert scale, ranging from very unimportant (1) to very important (5), by answering the following question: "How important is each specific statement in predicting a relapse in physical activity and/or dietary behavior?".

The on-site concept mapping session of the health practitioners lasted 1.5 hour and the session of the key population two hours. The difference in sorting and rating methods between stakeholder groups (online vs print) was based on recommendations of the health practitioners, who believed the online sorting of statements would be more comprehensible on paper. As participants came up with new 
statements during the brainstorm session, a portable label printer was used to print new cards for the key population. For the health practitioners, all new statements were directly entered in the online software. After the key population sessions, one of the researchers (ER) entered the data in Ariadne. Once entered in Ariadne, data could no longer be traced back to individuals.

\section{Step 4. Representation of statements}

Using the Ariadne-software, the structured statements were per subgroup transformed into a matrix representing the similarity between statements for each participant. A higher number signifies a higher conceptual similarity between ideas. All the individual matrices were then transformed into one matrix representing all the individuals in that subgroup. This matrix was used as input for the Principal Component Analysis (PCA), which translated the distance (i.e. correlations) between statements into coordinates in a multi-dimensional space (18). The statements were further classified by completing a cluster analysis with the coordinates of the statements. These clusters were graphically presented in a PCA plot. Statements that were closer to each other in the plot were sorted together more often (and vice versa). Additionally, the mean importance for each statement and cluster was calculated.

\section{Step 5. Interpretation of the maps}

By default Ariadne computed eight clusters. The research team discussed if more or less clusters represented participant's statements better, by evaluating the coherence between statements in each cluster. After defining the final number of clusters, each statement within a cluster was evaluated and allocated to a perceived predictor (e.g. the statement "lack of motivation" was allocated to the perceived predictor "motivation"). Subsequently, the research team named all clusters considering the names given by the participants. Within the groups, each cluster represented multiple perceived predictors; therefore it was not possible to do a group comparison on cluster level. Hence, we analyzed our results on predictor level instead of cluster level, which is in accordance with former concept mapping literature (19). To do so, the mean importance of each perceived predictor was calculated based on the overall mean importance ratings of the underlying statements. A perceived predictor was indicated as 'important' when the mean importance rating score was $>4.0$.

\section{Results}

\section{Participants}

A total of 39 health practitioners participated in this study (39 women, Mage $=48.2$ years, SDage $=9.9$ ), distributed over five groups, see Table 1. For the key population, 21 participants participated in this study (18 women, Mage $=53.5$ years, SDage $=8.9)$, distributed over three groups, see Table 1 . For both stakeholder groups saturation was reached, meaning no new statements came up during the last concept mapping sessions. 
Table 1

Demographics and characteristics of health practitioners and the key population.

Health practitioners

$(\mathrm{N}=39)$

Gender ( $N)$

Male

0

39
Key population

$(\mathrm{N}=21)$
$48.2(9.9)$

3

12

24

14

Profession $(\mathrm{N})$

Dietitian

Weight loss consultant

Lifestyle coach

Other

* Low level of education: primary education and lower general secondary education; Middle level of education: higher general secondary education, pre-university education, and secondary vocational education; High level of education: bachelor's degree, master's degree, and doctoral degree.

\section{Health practitioners}

The subgroups of health practitioners produced between 66 and 79 unique statements, sorted in 38 different predictors, including 32 individual predictors and six environmental predictors, see Supplementary file 2. There were 17 recurrent (mentioned in all health practitioner groups) perceived predictors of relapse in physical activity and dietary behavior: change in daily structure; stress; lack of effective coping skills; habitual behavior; lack of self-efficacy regarding losing weight; decrease in motivation; lifestyle imbalance or experiencing a life event; lack of perseverance; negative emotional state; non-supportive physical environment; goal disengagement; lack of social support; non-effective weight loss strategy; obstructing beliefs about lifestyle change; abstinence violation effect; perceived financial barriers; and perceived weather barriers.

Of the recurrent perceived predictors, five predictors were rated as important, i.e. mean rating $>4.0$, by all health practitioner groups: change in daily structure, stress, lack of effective coping skills, habitual 
behavior, and lack of self-efficacy regarding losing weight.

\section{Key population}

The subgroups of the key population produced between 35 and 81 statements, sorted in 31 different predictors, including 26 personal and five environmental predictors, see Supplementary file 2 . There were 13 recurrent (mentioned in all key population groups) perceived predictors of relapse in physical activity and dietary behavior: obstructing beliefs about lifestyle change; negative emotional state; indulgence; lack of knowledge; lifestyle imbalance or experiencing a life event; decrease in motivation; lack of perseverance; physiological barriers; perceived time barriers; perceived weather barriers; tempting social environment; lack of social support; and abstinence violation effect.

Of the recurrent perceived predictors, none were rated as important, i.e. mean rating $>4.0$, by all key population groups. The six recurrent perceived predictors with the highest rating scores (between 3.7 and 3.9) were: lifestyle imbalance or experiencing a life event, lack of perseverance, negative emotional state, abstinence violation effect, decrease in motivation, and indulgence.

\section{Discussion}

With this concept mapping study we aimed to identify predictors of relapse in physical activity and dietary behavior from the perspectives of health practitioners and the key population. The majority of perceived predictors were mentioned by both health practitioners and the key population, and the great majority of perceived predictors belonged to the individual domain. However, the predictors that were rated as most important differed between the two groups of stakeholders. According to the health practitioners, the important predictors were: change in daily structure, stress, lack of effective coping skills, habitual behavior, and lack of self-efficacy regarding losing weight. The key population groups rated none of the recurrent perceived predictors as important $(>4.0)$, however recurrent perceived predictors with the highest rating scores (between 3.7 and 3.9) were: lifestyle imbalance or experiencing a life event, lack of perseverance, negative emotional state, abstinence violation effect, decrease in motivation and indulgence.

The majority of our results is coherent with findings from quantitative relapse studies. A recent systematic review of prospective studies on predictors of relapse in dietary behavior and physical activity confirmed self-efficacy and coping skills as predictors (11). The abstinence violation effect has also been confirmed as predictor in the past (20-25). Besides, the majority of the perceived predictors rated as most important are also covered in the Relapse Prevention Model (RPM) (4), including stress, lifestyle imbalance or experiencing a life event, indulgence, negative emotional state, coping skills, self-efficacy, and the abstinence violation effect.

However, although covered in the RPM, for stress, negative emotional state, indulgence, and lifestyle imbalance or experiencing a life event, who were rated as most important by our stakeholders, further scientific evidence on their relation with relapse in physical activity and dietary behavior is lacking (11). 
Previous research on factors influencing (maintenance of) physical activity and dietary behavior may provide some first indications. For example, recent reviews showed that stress impairs efforts to be physically active, and stress and negative emotions unfavorably influences dietary behavior (26-29); however, evidence for an association between negative emotions and physical activity remains inconclusive $(30,31)$. Furthermore, indulgence has been associated with unhealthy eating behavior by multiple studies, especially when the indulgence is justified ("I worked so hard today, I deserve it") (32, 33). Despite the indicated association between lifestyle imbalance and relapse according to the RPM, studies assessing this potential association are, to our knowledge, currently lacking.

Although not part of the RPM, our stakeholders also indicated decrease in motivation, habitual behavior, change of daily rhythm and lack of perseverance as most important perceived predictors. As again scientific evidence on their association with relapse in physical activity and dietary behavior is lacking (11), general theories on motivation and behavior change and research on factors influencing (maintenance of) physical activity and dietary behavior may provide more insight. First, the selfdetermination theory indicates intrinsic motivation as an important factor for behavior change maintenance: behavior change is more likely to be maintained if the new behavior is perceived as personally relevant and reflects an individual's values (34). As most individuals start behavior change attempts when their motivation is high, a decrease in motivation over time could lead to a relapse into previous behavior (34). The association between intrinsic motivation and health behavior change has been shown present in the area of physical activity and dietary behavior (35-37). Second, habits, defined as a form of automaticity that is triggered by situational cues and enacted with little conscious awareness, play an important role in people's failure to adopt and maintain healthy behavior $(38,39)$. Therefore, both breaking and creating habits are central to behavior change (39). Especially eating habits are striking: research shows eating habits can be directly activated by environmental cues, without the activation of preferences and goals $(40,41)$. The formation of new habits is triggered by stable features in the environment (42); therefore, it may explain why a change in an individual's daily rhythm, often related to change of environment (e.g. going on holidays), can lead to a relapse into previous behavior. Last, for perseverance, described as the continuation of a goal-directed action in spite of obstacles (43), knowledge on the potential association with behavior change is currently lacking. More research is recommended to confirm the relation between these perceived predictors and relapse in physical activity and dietary behavior.

Although the majority of perceived predictors were mentioned by both stakeholder groups, both groups had somewhat different opinions. Different perceived predictors were rated as most important according to the health practitioners, when compared to the key population. Also, a few perceived predictors were mentioned by all practitioner groups, but not by the key population: change in daily structure, perceived financial barriers, and habitual behavior. Vice versa, perceived predictors that were mentioned by all key population groups, but not by the health practitioners were: tempting social environment, indulgence, and lack of knowledge. The differences may be due to health practitioners basing their knowledge on their experience with many clients, and therefore generate and rate statements on the average person (seeing 'the bigger picture'). The key population may have generated and rated statements based on their own 
experiences, leaving more room for diversity. This emphasizes the importance of including multiple stakeholders to gather diverse views and a more complete picture. Furthermore, results show that both stakeholder groups predominantly rate individual factors as most important perceived predictors of relapse. However, previous research indicates that environmental factors, such as an tempting environment, also influence relapse (11). A possible explanation for this difference might be that individuals do not know or like to admit that they are being influenced by their social or physical environment. Also, the influence of the social or physical environment is often through individual factors (e.g. not being able to cope with the social pressure at a party), which might make individual factors more proximal and therefore easier to recall. It may also be that more individual factors are mentioned due to the current stigma that overweight and obese individuals are to blame for their weight (44).

\section{Strengths and limitations}

Our study provides new insights into the predictors of relapse in physical activity and dietary behavior from the perspective of key stakeholders, contributing to a more in-depth understanding of relapse by means of personal perspectives and daily practice. The focus on both health practitioners and key population perspectives is an important strength of this study, providing insight from multiple points of view. Furthermore, the concept mapping method allows multiple points of view in each group of stakeholders to be integrated whilst taking the relative importance of each statement into account, using valid statistical methods (45). However, there are also some limitations worth mentioning. During the sorting of the statements we noticed that both stakeholder groups found it challenging to start sorting the statements into piles. This was mainly since they were not allowed to create more than 10 piles, due to the settings of the software program. As the stakeholders identified a wide range of predictors, it might have been easier to place the statements into better fitting categories if they were allowed to create more piles. Furthermore, as each cluster represented multiple perceived predictors, which appeared during the interpretation of the maps, it seems that the data may have been too complex to base the results entirely on an mathematical model. Therefore, expert opinion and existing theoretical categories were needed to be able to analyze the results on a predictor level instead of cluster level. Last, a more diverse sample would have been preferred: males were underrepresented and the majority of the key population had a middle educational level.

\section{Future research}

Several recommendations for future research can be made. First, as we wanted to keep the generation of statements feasible and non-confusing for the participants, we formulated one focus statement in which the predictors of physical activity and dietary behavior were combined and no distinction between lapse and relapse was made. Future research could investigate potential differences between the predictors of relapse in physical activity and dietary behavior, and between lapse and relapse. Second, for several predictors only studies that examined their association with general behavior change instead of relapse have been conducted. Therefore, to examine whether the identified perceived predictors indeed predict relapse in physical activity and dietary behavior, a larger prospective study is recommended. We suggest 
an ecological momentary assessment (EMA) study to track experiences over time and get insight into the process of behavior change, among which lapsing and relapsing (46). EMA has been proven useful in measuring lapses and relapses in previous studies (47-52), and therefore provides an opportunity to confirm the perceived predictors as identified in this study.

\section{Practical implications}

Although more research on the predictors of relapse in physical activity and dietary behavior is recommended, careful implications for practice can be made. For example, the differences between health practitioners and key population regarding the perceived predictors in relapse may provide an opportunity to enhance lifestyle coaching, by ensuring it is patient-centered and tailored (53). Clients are more likely to be satisfied and follow advice on health behavior change when they feel they have been heard and understood, and are given information they recognize as relevant to them (54). The identified predictors could be relevant for future weight loss interventions that combine evidence-based techniques for altering relevant changeable predictors (e.g. effective coping skills) and coping with relevant nonchangeable predictors (e.g. experiencing a life event), to prevent relapse in physical activity and dietary behavior. Planning coping responses on anticipated personal high risk situations helps an individual to cope with difficult situations, such as dealing with negative emotions or being tempted by their social or physical environment (55). Coping planning has been showed as an efficacious technique for promoting health behavior change, especially when individuals are supported in forming coping plans (56). Therefore, we recommend health practitioners to support their clients by helping to identify personal risk situations and formulate corresponding coping plans.

\section{Conclusion}

To conclude, our study provides new insights into the predictors of relapse in physical activity and dietary behavior identified as relevant by health practitioners (i.e. change in daily structure, stress, lack of effective coping skills, habitual behavior, and lack of self-efficacy) as well as the key population (i.e. lifestyle imbalance, experiencing a life event, lack of perseverance, negative emotional state, abstinence violation effect, decrease in motivation and indulgence). Both stakeholder groups predominantly rated individual factors as most the important perceived predictors of relapse. Our finding that both groups identified some different predictors or differed in opinion regarding the importance of the perceived predictors may provide an opportunity to enhance lifestyle coaching, by ensuring it is patient-centered and tailored.

\section{Abbreviations}

RPM

Relapse Prevention Model

CBS

Central Bureau of Statistics Netherlands 
PCA

Principal Component Analysis

EMA

Ecological Momentary Assessment

\section{Declarations}

\section{Ethics approval and consent to participate}

According to the Dutch Medical Research Involving Human Subjects Act, our study did not require further evaluation by the Research Ethics Review Committee. Online informed consent was obtained from all study participants.

\section{Consent for publication}

Not applicable.

\section{Availability of data and materials}

The datasets used and analyzed during the current study are available from the corresponding author on reasonable request.

\section{Competing interests}

The authors declare that they have no competing interests.

\section{Funding}

The contribution of ER and MVS was supported by the Innovational Research Incentives Scheme Veni from NWO-SSH (Netherlands Organisation for Scientific Research-Division for Social Sciences and Humanities) under project number 451-16-018.

\section{Authors' contributions}

ER contributed to the design of the study, data collection and analysis, interpretation of data, and drafting of the manuscript. MVS and WK contributed to the design of the study, data collection, interpretation of the data, and revising the manuscript. IS contributed to the design of the study, interpretation of data, and revising the manuscript. $\mathrm{MC}$ contributed to the design of the study and revising the manuscript. All authors read and approved the final manuscript. 


\section{Acknowledgements}

Not applicable.

\section{References}

1. Magkos F, Fraterrigo G, Yoshino J, Luecking C, Kirbach K, Kelly SC, et al. Effects of moderate and subsequent progressive weight loss on metabolic function and adipose tissue biology in humans with obesity. Cell Metabol. 2016;23(4):591-601.

2. Turk MW, Yang K, Hravnak M, Sereika SM, Ewing LJ, Burke LE. Randomized clinical trials of weightloss maintenance: A review. J Cardiovasc Nurs. 2009;24(1):58.

3. Marlatt GA, George WH. Relapse prevention: Introduction and overview of the model. British journal of addiction. 1984;79(4):261-73.

4. Marlatt G, Gordon J. Relapse prevention: Maintenance strategies in the treatment of addictive behaviors. New York: Guilford; 1985.

5. Baumeister RF, Heatherton TF, Tice DM. Losing control: How and why people fail at self-regulation. San Diego: Academic Press; 1994.

6. Marlatt GA, Donovan DM. Relapse prevention: Maintenance strategies in the treatment of addictive behaviors: Guilford press; 2005.

7. Dombrowski SU, Sniehotta FF, Avenell A, Johnston M, MacLennan G, Araújo-Soares V. Identifying active ingredients in complex behavioural interventions for obese adults with obesity-related comorbidities or additional risk factors for co-morbidities: a systematic review. Health Psychology Review. 2012;6(1):7-32.

8. Larimer ME, Marlatt GA. Relapse prevention: An overview of Marlatt's cognitive-behavioral model. Psychosocial Treatments: Routledge; 2004. pp. 11-28.

9. Baumeister RF, Heatherton TF. Self-regulation failure: An overview. Psychological inquiry. 1996;7(1):1-15.

10. Baumeister RF, Schmeichel BJ, Vohs KD. Self-regulation and the executive function: The self as controlling agent. Social psychology: Handbook of basic principles. 2007;2:516-39.

11. Roordink E, Steenhuis I, Kroeze W, Schoonmade L, Sniehotta F, van Stralen M. Predictors of Lapse and Relapse in Physical Activity and Dietary Behavior: A Literature Review on Prospective Studies.

12. Burke JG, O'Campo P, Peak GL, Gielen AC, McDonnell KA, Trochim WM. An introduction to concept mapping as a participatory public health research method. Qual Health Res. 2005;15(10):1392-410.

13. Trochim W, Kane M. Concept mapping: an introduction to structured conceptualization in health care. Int J Qual Health Care. 2005;17(3):187-91.

14. Trochim WM. An introduction to concept mapping for planning and evaluation. Eval Program Plan. 1989;12(1):1-16. 
15. Kane M, Trochim WM. Concept mapping for planning and evaluation: Sage Publications Thousand Oaks, CA; 2007.

16. Netherlands CBoS. Classificaties n.d. [Available from: https://www.cbs.nl/nl-nl/onzediensten/methoden/classificaties.

17. Ariadne. Ariadne for concept mapping online n.d. [Available from: http://www.minds21.org/.

18. Sleddens EF, Kroeze W, KohI LF, Bolten LM, Velema E, Kaspers P, et al. Correlates of dietary behavior in adults: an umbrella review. Nutrition reviews. 2015;73(8):477-99.

19. Hidding $L$, Chinapaw $M$, Altenburg T. An activity-friendly environment from the adolescent perspective: a concept mapping study. International Journal of Behavioral Nutrition Physical Activity. 2018;15(1):99.

20. Herman CP, Mack D. Restrained and unrestrained eating 1. Journal of personality. 1975;43(4):64760.

21. Polivy J, Herman CP, Younger JC, Erskine B. Effects of a model on eating behavior: The induction of a restrained eating style 1. J Pers. 1979;47(1):100-17.

22. Polivy J. Perception of calories and regulation of intake in restrained and unrestrained subjects. Addict Behav. 1976;1(3):237-43.

23. Carels RA, Hoffman J, Collins A, Raber AC, Cacciapaglia H, O'Brien WH. Ecological momentary assessment of temptation and lapse in dieting. Eat Behav. 2001;2(4):307-21.

24. Kendzierski D, Sheffield A. Self-schema and attributions for an exercise lapse. Basic Appl Soc Psychol. 2000;22(1):1-8.

25. Stetson BA, Beacham AO, Frommelt SJ, Boutelle KN, Cole JD, Ziegler CH, et al. Exercise slips in highrisk situations and activity patterns in long-term exercisers: An application of the relapse prevention model. Ann Behav Med. 2005;30(1):25-35.

26. Araiza AM, Lobel M. Stress and eating: Definitions, findings, explanations, and implications. Soc Pers Psychol Compass. 2018;12(4):e12378.

27. Stults-Kolehmainen MA, Sinha R. The effects of stress on physical activity and exercise. Sports medicine. 2014;44(1):81-121.

28. Frayn M, Knäuper B. Emotional eating and weight in adults: a review. Current Psychology. 2018;37(4):924-33.

29. Devonport TJ, Nicholls W, Fullerton C. A systematic review of the association between emotions and eating behaviour in normal and overweight adult populations. Journal of Health Psychology. 2019;24(1):3-24.

30. Liao Y, Shonkoff ET, Dunton GF. The acute relationships between affect, physical feeling states, and physical activity in daily life: a review of current evidence. Front Psychol. 2015;6:1975.

31. Kruk M, Zarychta K, Horodyska K, Boberska M, Scholz U, Radtke T, et al. What comes first, negative emotions, positive emotions, or moderate-to-vigorous physical activity? Mental Health Physical Activity. 2019;16:38-42. 
32. Prinsen S, Evers C, de Ridder D. Oops I did it again: Examining self-licensing effects in a subsequent self-regulation dilemma. Applied Psychology: Health Well-Being. 2016;8(1):104-26.

33. Effron DA, Monin B, Miller DT. The unhealthy road not taken: Licensing indulgence by exaggerating counterfactual sins. J Exp Soc Psychol. 2013;49(3):573-8.

34. Kwasnicka D, Dombrowski SU, White M, Sniehotta F. Theoretical explanations for maintenance of behaviour change: a systematic review of behaviour theories. Health psychology review. 2016;10(3):277-96.

35. Ng JY, Ntoumanis N, Thøgersen-Ntoumani C, Deci EL, Ryan RM, Duda JL, et al. Self-determination theory applied to health contexts: A meta-analysis. Perspectives on Psychological Science. 2012;7(4):325-40.

36. Amireault S, Godin G, Vézina-Im L-A. Determinants of physical activity maintenance: a systematic review and meta-analyses. Health Psychology Review. 2013;7(1):55-91.

37. Teixeira PJ, Carraça EV, Markland D, Silva MN, Ryan RM. Exercise, physical activity, and selfdetermination theory: a systematic review. International journal of behavioral nutrition physical activity. 2012;9(1):78.

38. Orbell S, Verplanken B. The automatic component of habit in health behavior: Habit as cuecontingent automaticity. Health psychology. 2010;29(4):374.

39. Wood W, Neal DT. Healthy through habit: Interventions for initiating \& maintaining health behavior change. Behavioral Science Policy. 2016;2(1):71-83.

40. Rothman AJ, Sheeran P, Wood W. Reflective and automatic processes in the initiation and maintenance of dietary change. Ann Behav Med. 2009;38(suppl_1):s4-17.

41. Neal DT, Wood W, Wu M, Kurlander D. The pull of the past: When do habits persist despite conflict with motives? Pers Soc Psychol Bull. 2011;37(11):1428-37.

42. Wood W, Quinn JM, Kashy DA. Habits in everyday life: Thought, emotion, and action. J Personal Soc Psychol. 2002;83(6):1281.

43. Peterson C, Seligman ME. Character strengths and virtues: A handbook and classification: Oxford University Press; 2004.

44. Puhl RM, Heuer CA. Obesity stigma: important considerations for public health. American journal of public health. 2010;100(6):1019-28.

45. Rosas SR, Kane M. Quality and rigor of the concept mapping methodology: a pooled study analysis. Eval Program Plan. 2012;35(2):236-45.

46. Shiffman S, Stone AA, Hufford MR. Ecological momentary assessment. Annu Rev Clin Psychol. 2008;4:1-32.

47. Carels RA, Cacciapaglia HM, Rydin S, Douglass OM, Harper J. Can social desirability interfere with success in a behavioral weight loss program? Psychology Health. 2006;21(1):65-78.

48. Carels RA, Douglass OM, Cacciapaglia HM, O'Brien WH. An ecological momentary assessment of relapse crises in dieting. J Consult Clin Psychol. 2004;72(2):341. 
49. Carels RA, Hoffman J, Collins A, Raber AC, Cacciapaglia H, O'Brien WH. Ecological momentary assessment of temptation and lapse in dieting. Eat Behav. 2001;2(4):307-21.

50. Forman EM, Schumacher LM, Crosby R, Manasse SM, Goldstein SP, Butryn ML, et al. Ecological momentary assessment of dietary lapses across behavioral weight loss treatment: characteristics, predictors, and relationships with weight change. Ann Behav Med. 2017;51(5):741-53.

51. Latner JD, McLeod G, O'Brien KS, Johnston L. The role of self-efficacy, coping, and lapses in weight maintenance. Eating and Weight Disorders-Studies on Anorexia. Bulimia Obesity. 2013;18(4):35966.

52. McKee HC, Ntoumanis N, Taylor IM. An ecological momentary assessment of lapse occurrences in dieters. Ann Behav Med. 2014;48(3):300-10.

53. Sladdin I, Ball L, Bull C, Chaboyer W. Patient-centred care to improve dietetic practice: an integrative review. Journal of human nutrition dietetics. 2017;30(4):453-70.

54. Gable J. Counselling skills for dietitians: Blackwell Publishing Ltd; 2007.

55. Sniehotta FF, Schwarzer R, Scholz U, Schüz B. Action planning and coping planning for long-term lifestyle change: theory and assessment. European Journal of Social Psychology. 2005;35(4):56576.

56. Kwasnicka D, Presseau J, White M, Sniehotta FF. Does planning how to cope with anticipated barriers facilitate health-related behaviour change? A systematic review. Health psychology review. 2013;7(2):129-45.

\section{Figures}


Health practitioners

Key population

\begin{tabular}{|c|c|c|}
\hline Step 1 & $\frac{\text { Preparation }}{\text { - Participants and recruitment }}$ & $\frac{\text { Preparation }}{\text { - Participants and recruitm ent }}$ \\
\hline \multirow{4}{*}{ Step 2} & \multicolumn{2}{|c|}{$\begin{array}{l}\text { Online } \\
\text { One week prior to on-site concept mapping session }\end{array}$} \\
\hline & $\frac{\text { Generation of statements }}{\text { - Individual brainstorm }}$ & $\frac{\text { Generation of statements }}{\text { - Individual brainstorm }}$ \\
\hline & \multicolumn{2}{|c|}{ On-site concept mapping session } \\
\hline & $\begin{array}{l}\text { Generation of statements } \\
\text { - Group brainstorm } \\
\text { - Input from individual brainstorm }\end{array}$ & $\begin{array}{l}\text { Generation of statements } \\
\text { - Group brainstorm } \\
\text { - Input from individual brainstorm }\end{array}$ \\
\hline & Structuring of statements (indiv idual) & Structuring of statem ents (indiv idual) \\
\hline Step 3 & $\begin{array}{l}\text { - Sorting of statements-laptop } \\
\text { - Rating of statem ents-laptop }\end{array}$ & $\begin{array}{l}\text { - Sorting of statements-paper } \\
\text { - Rating of statements-paper }\end{array}$ \\
\hline Step 4 & $\frac{\text { Representation of statements }}{\text { - Com putation of } m \text { aps }}$ & $\frac{\text { Representation of statements }}{\text { - Com putation of } \mathrm{m} \text { aps }}$ \\
\hline & $\frac{\text { Interpretation of maps }}{\text { - Statem ent lists }}$ & $\frac{\text { Interpretation of maps }}{\text { - Statem ent lists }}$ \\
\hline Step 5 & $\begin{array}{l}\text { - Cluster lists } \\
\text { - Allocation statements to } \\
\text { predictor } \\
\text { - Final concept maps }\end{array}$ & $\begin{array}{l}\text { - Cluster lists } \\
\text { - Allocation statements to } \\
\text { predictor } \\
\text { - Final concept maps }\end{array}$ \\
\hline
\end{tabular}

\section{Figure 1}

The concept mapping process. Adapted from "An introduction to concept mapping for program planning and evaluation" by W. Trochim, 1989, Evaluation and Program Planning, 12, 1-16.

\section{Supplementary Files}

This is a list of supplementary files associated with this preprint. Click to download.

- BMCSupplementaryfile2.docx

- BMCSupplementaryfile1.docx 